\title{
Fluorescence in situ hybridization as a tool for microstaging in malignant melanoma
}

\author{
Marissa D Newman ${ }^{1}$, Terakeith Lertsburapa ${ }^{1}$, Marjan Mirzabeigi ${ }^{1}$, Mariam Mafee ${ }^{1}$, \\ Joan Guitart ${ }^{1}$ and Pedram Gerami ${ }^{1,2}$ \\ ${ }^{1}$ Department of Dermatology, The Feinberg School of Medicine, Northwestern University, Chicago, IL, USA \\ and ${ }^{2}$ Robert Lurie Cancer Center of Northwestern University, Chicago, IL, USA
}

\begin{abstract}
Up to $30-50 \%$ of melanomas arise in association with a nevus. Accurately defining, the nevus from the melanoma can significantly affect microstaging. Recently, we showed that a targeted fluorescence in situ hybridization (FISH) assay could distinguish between benign nevi and melanoma with a sensitivity of $87 \%$ and specificity of $95 \%$. In this study, we evaluated the potential of this same assay for use in the microstaging of melanoma. We performed FISH on 36 cases of melanoma occurring in association with a nevus and 6 cases of nevoid melanoma with deep dermal involvement. In the melanomas with associated benign nevi, FISH enumeration was performed separately on the histologically malignant and benign components. In the nevoid melanomas, FISH was performed on the deep dermal areas. On the basis of the criteria developed in our earlier studies, we determined the sensitivity of the assay within the malignant areas and the specificity within the benign areas of melanomas with associated nevi. In addition, we evaluated the sensitivity and specificity within a group of six nevoid melanomas with deep dermal involvement. Among melanomas with associated nevi, 28 of 36 cases $(78 \%)$ tested positively in the histologically malignant areas. The benign nevus components were uniformly negative for all criteria. Six of six nevoid melanomas (100\%) tested positively in the deep dermal area. FISH analysis with probes targeting 6 p25, 6q23, 11q13 and CEP6 can effectively discriminate the malignant and benign components of melanomas with associated nevi and can be used as an adjunctive tool for microstaging. The assay has high sensitivity for the malignant areas of nevus-associated melanomas and outstanding specificity for the benign areas. The sensitivity is independent of the morphological features, and the assay performs well in nevoid melanoma cases.
\end{abstract}

Modern Pathology (2009) 22, 989-995; doi:10.1038/modpathol.2009.72; published online 15 May 2009

Keywords: melanoma; nevoid melanoma; fluorescence in situ hybridization; chromosome 6; 6p; 6q

Cutaneous melanoma is one of the most aggressive cancers with increasing incidence worldwide. ${ }^{1}$ The American Cancer Society predicted 62480 new cases of invasive melanoma in the United States in 2008 and that 8420 people would die of melanoma within the year. ${ }^{2}$ Currently, Breslow's depth is the most significant prognostic factor from the primary skin lesion for cutaneous melanomas. Hence, an accurate assessment of the Breslow's depth is crucial to providing accurate prognostic information and determining management issues, such as margins of excision and whether or not to perform a sentinel node biopsy. ${ }^{3}$ It has been reported that up to $30-50 \%$ of cutaneous melanomas occur with an associated

Correspondence: Dr P Gerami, Department of Dermatology, The Feinberg School of Medicine, Northwestern University, 676 North St. Clair Street, Suite 1600, Chicago, IL 60611, USA.

E-mail: pgerami@nmff.org

Received 2 February 2009; revised 7 April 2009; accepted 16

April 2009; published online 15 May 2009 nevus. ${ }^{4-6}$ Hence in 2008, between 18744 and 31240 cases of invasive melanoma were predicted to occur with an associated nevus., ${ }^{5,6}$ These numbers are likely underestimates because of under-reporting.

Determining an accurate Breslow's measurement can be especially difficult in cases of melanoma arising with an associated nevus. ${ }^{7}$ Independent of whether the initial precursor nevus is junctional or compound, over $99 \%$ of the melanomas begin at the dermal-epidermal junction before invading the dermis. Therefore, the deepest part of the neoplasm within the dermis frequently consists of a benign nevus. However, if the precursor nevus is dysplastic or shows significant atypia or pleomorphism, it can be extremely difficult to determine the line of transition between melanoma and nevus and, subsequently, the precise Breslow's depth. The measurement of Breslow's depth may be further complicated in cases of nevoid melanomas or in cases of melanoma with paradoxical maturation. In the latter, the deepest portion of the melanoma can 
show diminution in the size of nests and individual cells, thereby mimicking the pattern of a benign nevus. ${ }^{8}$

We have shown earlier that a fluorescence in situ hybridization (FISH) assay targeting 6p25 (RREB1), 6q23 (MYB), 11q13 (CCND1) and CEP6 could distinguish benign and malignant melanocytic neoplasms with high sensitivity and specificity. ${ }^{9}$ In this study, we evaluated 36 melanomas with associated nevi and 6 nevoid melanomas with deep dermal involvement using FISH with probes targeting $6 \mathrm{p} 25$, 6q23, CEP6 and 11q13. Using criteria that we have established earlier and optimized for distinguishing benign and malignant melanocytic neoplasms with this probe set, our goal was to evaluate (1) the percentage of melanomas with associated nevi testing positively with this test, (2) the utility of this assay in evaluating nevoid melanomas and (3) the utility of FISH targeting the above loci as a tool for microstaging in melanoma to improve the accuracy of the Breslow's assessment.

\section{Materials and methods}

After obtaining approval from the IRB and Northwestern University Cancer Center, we reviewed our dermatopathology files at Northwestern University for cases of melanoma arising within a precursor nevus and cases of nevoid melanomas. For cases of melanoma with associated nevi, we selected only cases in which there was no histological ambiguity and in which well-defined benign and malignant components could be identified in the same sections. In many cases, the histological findings were those of a compound melanocytic neoplasm, with the junctional component consisting primarily of melanoma in situ and the dermal component being a benign precursor or coexisting nevus. Some cases with invasive melanoma were also used in which the benign component was sufficiently distinct in its position in the sections, allowing it to be easily identifiable in FISH sections. Thirty-six cases from 2000 to 2008 met these criteria. Table 1 contains demographic information including the age and sex of the patient in addition to the site, histological subtype and Breslow's depth of the melanoma. Six cases of nevoid melanomas with deep dermal involvement were also evaluated (Table 2).

Probes targeting four loci were used for FISH evaluation on the basis of the results of our earlier study. ${ }^{9}$ These included Ras Responsive ElementBinding Protein-1 (VysisLSI RREB1-Spectrum Red), myeloblastosis (VysisLSI MYB-S Gold), cyclin D1 or chromosome 11q (VysisLSI CCND1Spectrum Green) and centromeric enumeration probe control for chromosome 6 (VysisLSI CEP6Spectrum Aqua) from Abbott Molecular Inc. (Des Plaines, IL, USA).
Table 1 Demographic information and classification for melanomas with associated nevi

\begin{tabular}{|c|c|c|c|c|c|}
\hline Subject & Age & Sex & Site & Subtype & $\begin{array}{c}\text { Breslow's } \\
\text { depth (mm) }\end{array}$ \\
\hline 1 & 62 & M & Tragus & SS & 0.43 \\
\hline 2 & 60 & M & Forearm & SS & 0 \\
\hline 3 & 72 & M & Back & SS & 1.16 \\
\hline 4 & 51 & M & Forearm & SS & 0.74 \\
\hline 5 & 66 & $\mathrm{~F}$ & Leg & SS & 0.75 \\
\hline 6 & 50 & M & Leg & SS & 0.23 \\
\hline 7 & 44 & M & Chest & SS & 1.3 \\
\hline 8 & 49 & M & Back & SS & 0.67 \\
\hline 9 & 26 & $\mathrm{M}$ & Chest & SS & 0 \\
\hline 10 & 56 & $\mathrm{~F}$ & Forearm & SS & 0 \\
\hline 11 & 40 & $\mathrm{~F}$ & Scapula & SS & 0.6 \\
\hline 12 & 67 & $\mathrm{~F}$ & Forearm & SS & 0.35 \\
\hline 13 & 24 & $\mathrm{~F}$ & Back & SS & 0.32 \\
\hline 14 & 64 & M & Back & SS & 0 \\
\hline 15 & 30 & $\mathrm{~F}$ & Back & SS & 0 \\
\hline 16 & 31 & $\mathrm{~F}$ & Leg & SS & 1.27 \\
\hline 17 & 17 & M & Abdomen & SS & 0 \\
\hline 18 & 82 & M & Back & SS & 0 \\
\hline 19 & 44 & $\mathrm{~F}$ & Back & SS & 0 \\
\hline 20 & 60 & $\mathrm{~F}$ & Cheek & SS & 0.9 \\
\hline 21 & 24 & $\mathrm{~F}$ & Anterior neck & SS & 0.38 \\
\hline 22 & 71 & M & Back & SS & 0 \\
\hline 23 & 26 & M & Back & SS & 0.42 \\
\hline 24 & 44 & $\mathrm{~F}$ & Back & SS & 0 \\
\hline 25 & 62 & $\mathrm{~F}$ & Ankle & SS & 0.13 \\
\hline 26 & 58 & $\mathrm{M}$ & Back & SS & 0 \\
\hline 27 & 28 & $\mathrm{M}$ & Back & SS & 0 \\
\hline 28 & 30 & M & Back & SS & 0 \\
\hline 29 & 42 & M & Scalp vertex & SS & 0.8 \\
\hline 30 & 53 & M & Back & Nodular & 1.7 \\
\hline 31 & 27 & $\mathrm{~F}$ & Buttock & SS & 0 \\
\hline 32 & 18 & $\mathrm{~F}$ & Forearm & SS & 0 \\
\hline 33 & 72 & $\mathrm{~F}$ & Back & SS & 0 \\
\hline 34 & 40 & M & Back & SS & 0.28 \\
\hline 35 & 76 & M & Back & SS & 0.67 \\
\hline 36 & 67 & M & Back & SS & 0 \\
\hline
\end{tabular}

SS, superficial spreading.

FISH for formalin-fixed paraffin-embedded tissue was performed for all cases. ${ }^{9}$ Sections of $5 \mu \mathrm{m}$ thickness were mounted onto SuperFrost Plus positively charged slides (ThermoShandon, Pittsburgh, PA, USA), baked at $56^{\circ} \mathrm{C}$ overnight, deparaffinized, submersed in $1 \times$ SSC, $\mathrm{pH} 6.3$, at $80^{\circ} \mathrm{C}$ for $35 \mathrm{~min}$ and washed in water for $3 \mathrm{~min}$. After protease digestion (4 mg pepsin/ml $0.2 \mathrm{~N} \mathrm{HCl}$ ) at $37^{\circ} \mathrm{C}$ for $15 \mathrm{~min}$, sections were rinsed in water for $3 \mathrm{~min}$, passed through graded ethanol and dried. Hybridizations were carried out at $37^{\circ} \mathrm{C}$ for $16-18 \mathrm{~h}$ in an automated co-denaturation oven (HYBrite or ThermoBrite Denaturation/Hybridization System; Abbott Molecular Inc.) according to the manufacturer's instructions. Sections were placed in washing buffer $(2 \times \quad$ SSC/0.3\% NP40; Abbott Molecular Inc.) at room temperature for 2-10 min to remove the coverslips and then immersed in $73^{\circ} \mathrm{C}$ washing buffer for $2 \mathrm{~min}$, dried and mounted with DAPI I antifade solution (Abbott Molecular Inc.). 
Table 2 FISH data for melanomas associated with nevi

\begin{tabular}{|c|c|c|c|c|}
\hline Subject & $\begin{array}{c}\text { RREB1/CEP6 } \\
\text { gain }\end{array}$ & $\begin{array}{l}\text { RREB1 } \\
\text { gain }\end{array}$ & $\begin{array}{c}M Y B / C E P 6 \\
\text { loss }\end{array}$ & $\begin{array}{l}\text { CCND1 } \\
\text { gain }\end{array}$ \\
\hline 1 & 26/30 (0.87) & $22 / 30(0.73)$ & $4 / 30(0.13)$ & $0 / 30(0)$ \\
\hline 2 & $22 / 30(0.73)$ & 18/30 (0.60) & $6 / 30(0.20)$ & $2 / 30(0.07)$ \\
\hline 3 & $27 / 30(0.90)$ & $27 / 30(0.90)$ & $17 / 30(0.57)$ & $1 / 30(0.03)$ \\
\hline 4 & $22 / 30(0.73)$ & $29 / 30(0.97)$ & $10 / 30(0.33)$ & $23 / 30(0.77)$ \\
\hline 5 & 28/30 (0.93) & $23 / 30(0.77)$ & $21 / 30(0.70)$ & $2 / 30(0.07)$ \\
\hline 6 & 19/30 (0.63) & $18 / 30(0.60)$ & $6 / 30(0.20)$ & $5 / 30(0.17)$ \\
\hline 7 & 23/30 (0.77) & $22 / 30(0.73)$ & $3 / 30(0.10)$ & $4 / 30(0.13)$ \\
\hline 8 & 18/30 (0.60) & 15/30 (0.50) & $10 / 30(0.33)$ & $0 / 30(0)$ \\
\hline 9 & 20/30 (0.67) & $14 / 30(0.47)$ & $8 / 30(0.27)$ & $0 / 30(0)$ \\
\hline 10 & 23/30 (0.77) & $22 / 30(0.73)$ & $6 / 30(0.20)$ & $4 / 30(0.13)$ \\
\hline 11 & $18 / 30(0.6)$ & $25 / 30(0.83)$ & $15 / 30(0.50)$ & $13 / 30(0.44)$ \\
\hline 12 & $23 / 30(0.77)$ & $21 / 30(0.70)$ & $10 / 30(0.33)$ & $1 / 30(0.03)$ \\
\hline 13 & $16 / 30(0.53)$ & $12 / 30(0.40)$ & $4 / 30(0.13)$ & $8 / 30(0.27)$ \\
\hline 14 & $21 / 30(0.70)$ & 19/30 (0.63) & $9 / 30(0.30)$ & $7 / 30(0.23)$ \\
\hline 15 & $14 / 30(0.47)$ & 19/30 (0.63) & $7 / 30(0.23)$ & $2 / 30(0.07)$ \\
\hline 16 & $16 / 30(0.53)$ & $22 / 30(0.73)$ & $6 / 30(0.20)$ & $10 / 30(0.33)$ \\
\hline 17 & 19/30 (0.63) & $15 / 30(0.5)$ & $4 / 30(0.13)$ & $6 / 30(0.20)$ \\
\hline 18 & $22 / 30(0.73)$ & $16 / 30(0.53)$ & $7 / 30(0.23)$ & $1 / 30(0.03)$ \\
\hline 19 & $6 / 30(0.20)$ & 9/30 (0.30) & $6 / 30(0.20)$ & $16 / 30(0.53)$ \\
\hline 20 & $16 / 30(0.53)$ & $9 / 30(0.30)$ & $4 / 30(0.13)$ & $0 / 30(0)$ \\
\hline 21 & 19/30 (0.63) & $16 / 30(0.53)$ & $7 / 30(0.23)$ & $2 / 30(0.07)$ \\
\hline 22 & 19/30 (0.63) & $11 / 30(0.37)$ & $8 / 30(0.27)$ & $0 / 30(0)$ \\
\hline 23 & 20/30 (0.67) & $14 / 30(0.47)$ & $14 / 30(0.47)$ & $2 / 30(0.07)$ \\
\hline 24 & 20/30 (0.67) & $17 / 30(0.57)$ & $6 / 30(0.20)$ & $0 / 30(0)$ \\
\hline 25 & $16 / 30(0.53)$ & 9/30 (0.30) & $4 / 30(0.13)$ & $0 / 30(0)$ \\
\hline 26 & $13 / 30(0.44)$ & $12 / 30(0.40)$ & $9 / 30(0.30)$ & $0 / 30(0)$ \\
\hline 27 & $14 / 30(0.47)$ & $11 / 30(0.37)$ & $6 / 30(0.20)$ & $3 / 30(0.10)$ \\
\hline 28 & $21 / 30(0.70)$ & 20/30 (0.67) & $1 / 30(0.03)$ & $1 / 30(0.03)$ \\
\hline 29 & $8 / 30(0.27)$ & $6 / 30(0.2)$ & $5 / 30(0.17)$ & $4 / 30(0.13)$ \\
\hline 30 & $9 / 30(0.30)$ & $5 / 30(0.17)$ & $6 / 30(0.20)$ & $6 / 30(0.20)$ \\
\hline 31 & $12 / 30(0.40)$ & $7 / 30(0.23)$ & $6 / 30(0.20)$ & $0 / 30(0)$ \\
\hline 32 & $11 / 30(0.37)$ & $5 / 30(0.17)$ & $1 / 30(0.03)$ & $0 / 30(0)$ \\
\hline 33 & $5 / 30(0.17)$ & $1 / 30(0.03)$ & $3 / 30(0.10)$ & $0 / 30(0)$ \\
\hline 34 & $10 / 30(0.33)$ & $5 / 30(0.17)$ & $9 / 30(0.30)$ & $1 / 30(0.03)$ \\
\hline 35 & $16 / 30(0.53)$ & $4 / 30(0.13)$ & $5 / 30(0.17)$ & $0 / 30(0)$ \\
\hline 36 & $14 / 30(0.47)$ & $8 / 30(0.27)$ & $8 / 30(0.27)$ & $0 / 30(0)$ \\
\hline
\end{tabular}

Bolded if met criteria.

In each case, an H\&E-stained section from the block was obtained followed directly by an unstained section for FISH. Benign and malignant areas were mapped out on the H\&E sections. For cases of melanoma with associated nevi, a separate evaluation was performed on the benign and malignant components. For the cases of nevoid melanomas, the evaluation was performed on the deep dermal area.

The slides were analyzed with an epifluorescence microscope equipped with single band-pass filters (Abbott Molecular Inc.). The analyses were performed by both three trained technicians and two dermatopathologists. All analyses were performed blinded of the specimens' diagnoses. Tumor-bearing areas were localized using the DAPI filter at low magnification. The tumor area was then thoroughly inspected for the presence of nuclei harboring abnormal copy numbers of any probe. Areas with the most significant copy number changes were selected for enumeration. Wherever possible, three abnormal areas were selected, and within each area, 10 random nuclei were analyzed under high power ( $\times 60$ objective). To qualify, nuclei had to be nonoverlapping and harbor sufficiently bright signals. Nuclei that showed no signals for more than one probe were not analyzed. Samples in which less than 30 nuclei could be evaluated were excluded from the study.

Criteria for a positive FISH result included (1) gain in RREB1 relative to CEP6 greater than $55 \%$ or (2) gain in RREB1 greater than $29 \%$, (3) loss in $M Y B$ relative to CEP6 greater than $40 \%$ and (3) gain in CCND1 greater than $38 \%$. The sensitivity for the FISH technique was calculated in comparison with the gold standard of histological diagnosis for melanoma. For cases with an associated nevus, we qualitatively evaluated the benign areas, and enumeration was performed if any significant gains or losses were identified. We then determined the overall percentage of cases meeting each of the four criteria in the malignant areas as well as in the benign areas. In the nevoid melanoma cases, the evaluator was blinded to the histological diagnosis.

\section{Results}

Among the 36 patients with melanomas associated with nevi, the average age was 48.1 years with a range from 18 to 82 years (Table 1). The female-tomale ratio was 15:21. Twenty-three cases occurred on the trunk as compared with nine cases on the extremities and four head and neck cases. Thirtyfive cases were superficial spreading melanomas. The remaining case was a nodular melanoma (Figure 1).

In all, 28 of $36(78 \%)$ cases of melanomas associated with nevi met at least one of the four criteria for melanoma in the malignant area as reported in bold type (Table 2). All 28 cases met the criteria for either RREB1 gain or RREB1/CEP6 imbalance primarily as a result of RREB1 gain (Figures 2 and 3). Only four cases met criteria for loss in $M Y B / C E P 6$, and three cases met criteria for gain in CCND1. In all, 21 of the $36(58 \%)$ cases satisfied two criteria, whereas 5/36 (14\%) cases met three criteria in a single specimen. One of the 36 $(3 \%)$ cases satisfied all the four criteria studied.

In the benign areas, the FISH evaluation was unexceptionally negative (Figure 3). Hence, there was $100 \%$ specificity for evaluation of the benign nevus component.

Among the six cases of nevoid melanomas, the average age was 47.2 years, with a range from 38 to 75 years of age (Table 3 ). The female-to-male ratio was 4:2. All six cases were positive by at least one criterion (Table 4). Five of the six (83\%) nevoid melanoma cases met malignancy criteria for RREB1 (Figure 4), 4/6 (67\%) cases met criteria for RREB1/ CEP $6,2 / 6(33 \%)$ cases met criteria for gain in 

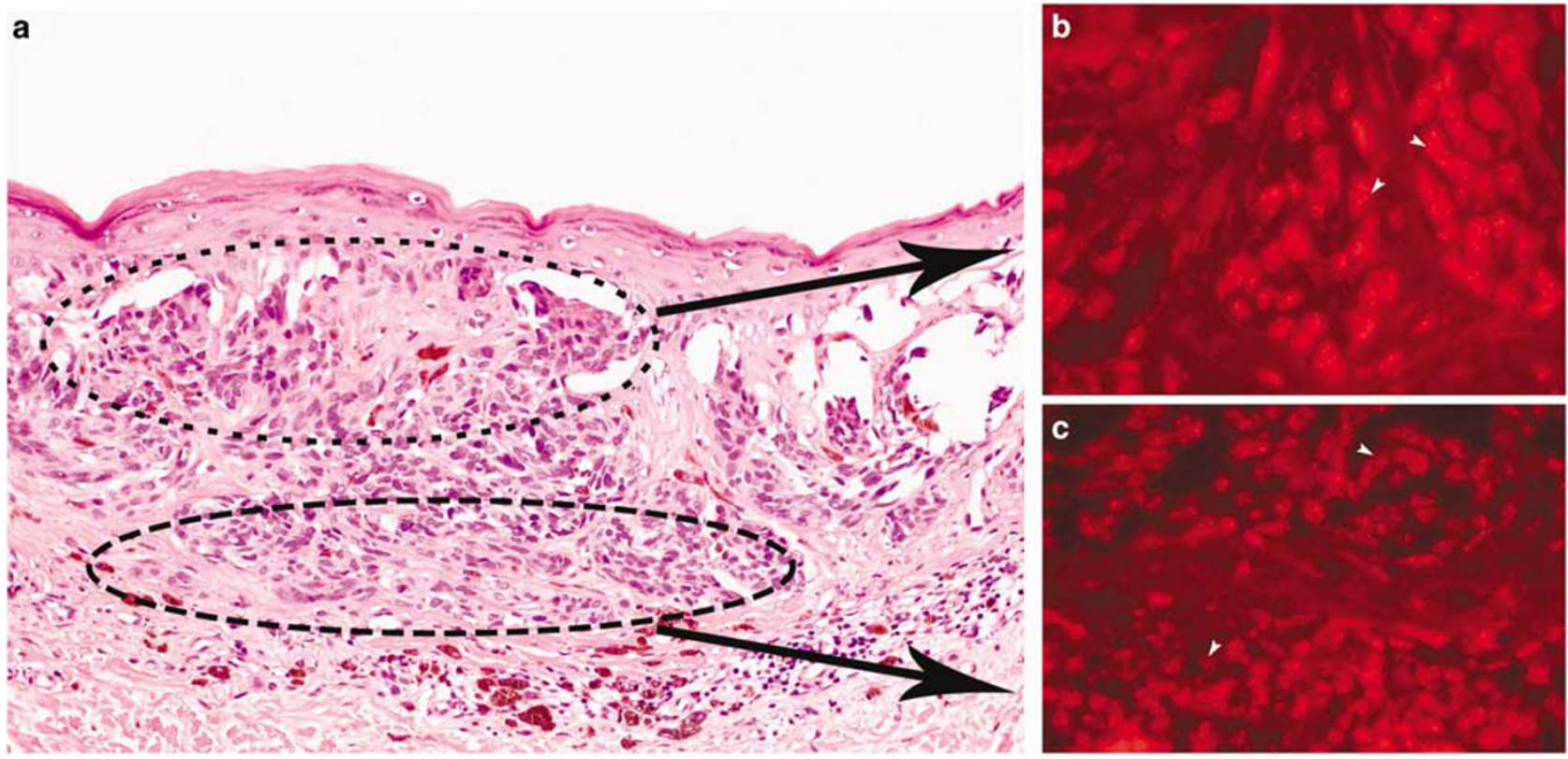

Figure 1 A representative low-power $(\mathbf{a} ; \mathrm{H} \& \mathrm{E}, \times 200)$ photograph showing expansile nests of malignant melanocytes at the dermalepidermal junction with a gradual transition to smaller nests and cells in the dermis. At higher power (b; FISH, $\times 600), 6 \mathrm{p}$ chromosomal gains are observed at the dermal-epidermal junction and $(\mathbf{c} ; \mathrm{FISH}, \times 600)$ nests from the superior aspect show $6 \mathrm{p}$ chromosomal gains, whereas deeper dermal nests show normal chromosomal copy numbers.

a
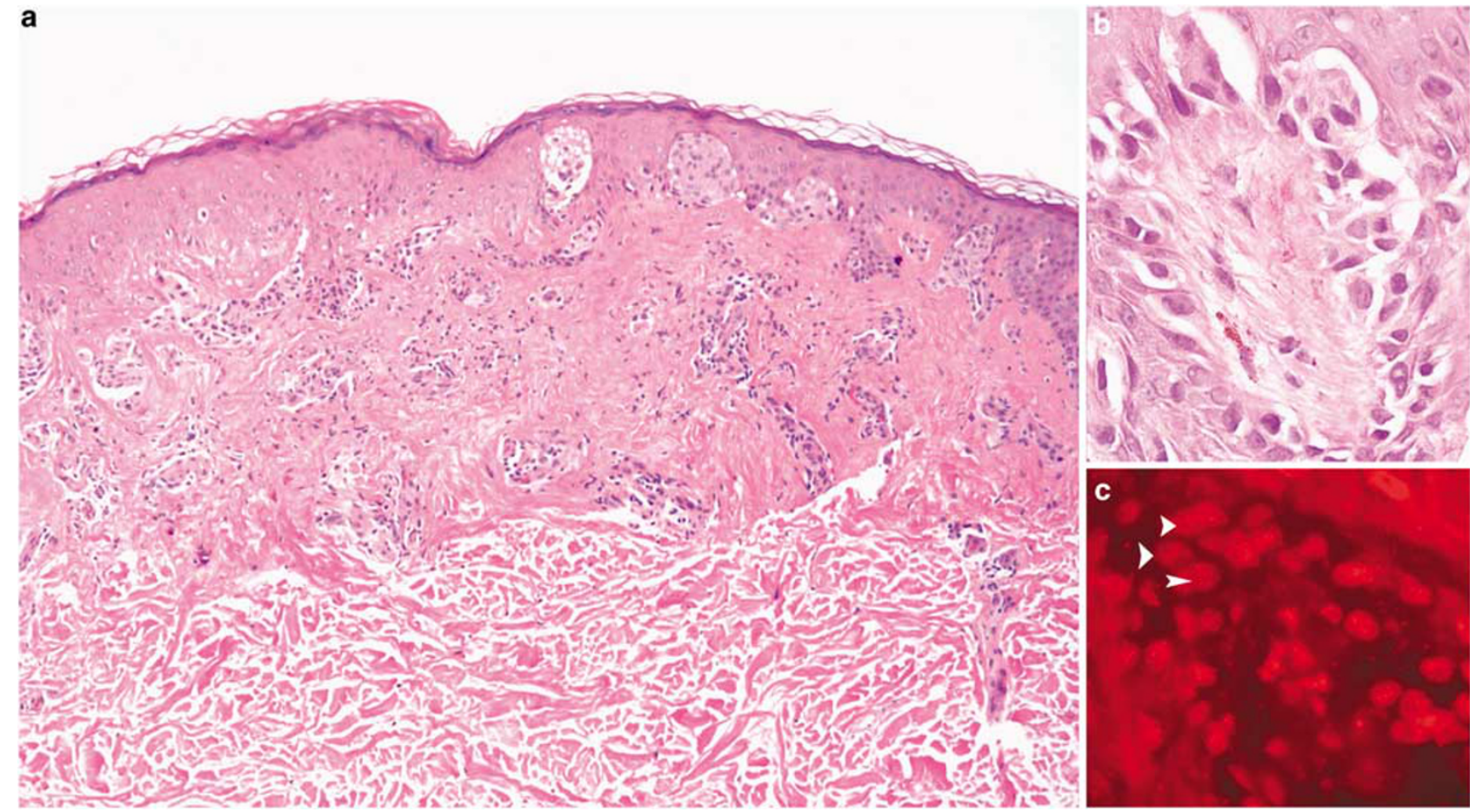

Figure 2 A representative low-power $(\mathbf{a} ; \mathrm{H \& E}, \times 100)$ photograph shows a melanoma in situ arising in a compound melanocytic neoplasm. At higher power $(\mathbf{b} ; \mathrm{H} \& \mathrm{E} \times 400)$, there are highly atypical melanocytes along the dermal-epidermal junction and $(\mathbf{c} ;$ FISH $\times 600) 6 \mathrm{p}$ chromosomal gains are observed, which correspond to photograph in $(\mathbf{b})$.

CCND1 and $1 / 6(17 \%)$ case met criteria for loss in $M Y B / C E P 6$. Four of the six (67\%) cases satisfied two criteria, whereas $2 / 6(33 \%)$ cases met three criteria in a single specimen.

\section{Discussion}

Fluorescence in situ hybridization is a new and emerging tool for diagnosis in melanocytic neo- 

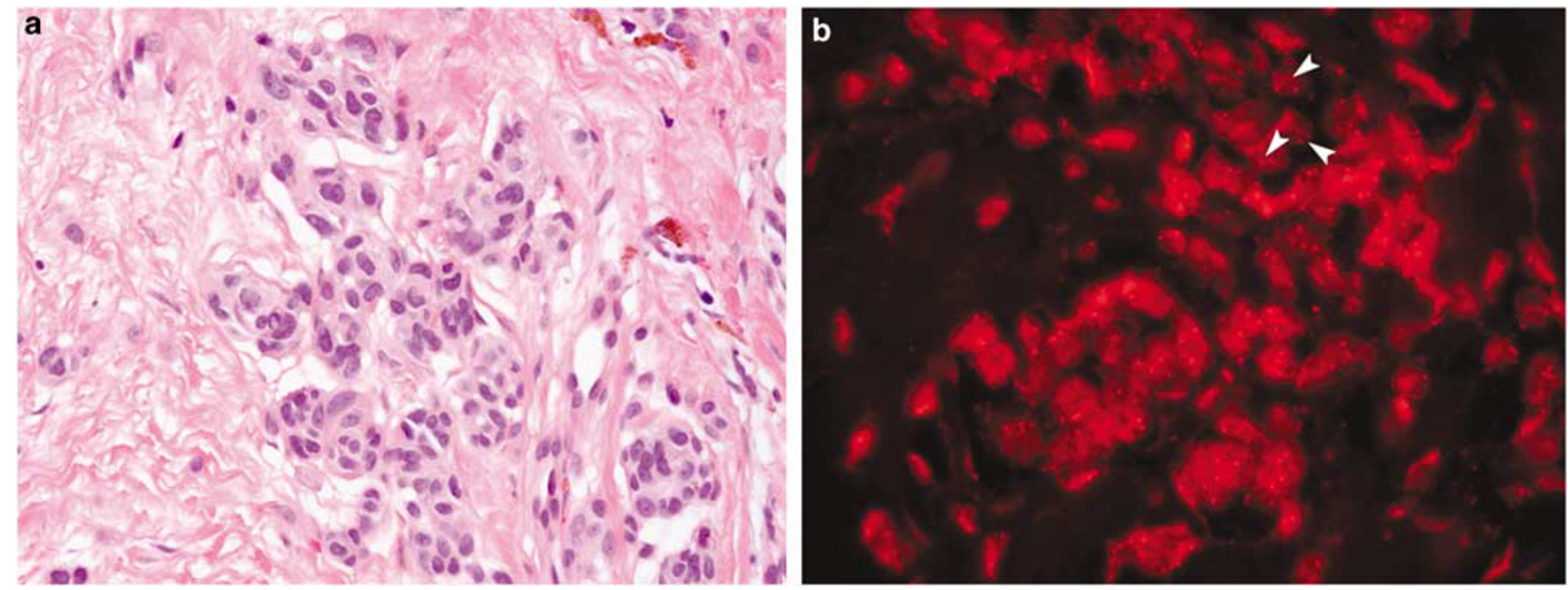

Figure 3 A representative high-power $(\mathbf{a} ; \mathrm{H} \& \mathrm{E}, \times 400)$ photograph shows benign melanocytes within the dermis and $(\mathbf{b} ;$ FISH, $\times 600)$ normal chromosomal copy numbers.

Table 3 Demographic information and classification of nevoid melanomas

\begin{tabular}{lccllc}
\hline Subject & Age & Sex & Site & Subtype & $\begin{array}{c}\text { Breslow's } \\
\text { depth (mm) }\end{array}$ \\
\hline 1 & 75 & M & Forehead & Nodular & 1.05 \\
2 & 41 & F & Shoulder & Nodular & 0.75 \\
3 & 39 & F & Thigh & Nodular & 1.2 \\
4 & 50 & F & Chest & Nodular & 0.55 \\
5 & 38 & M & Neck & Nodular & 1.0 \\
6 & 40 & F & Arm & Nodular & 0.75 \\
\hline
\end{tabular}

Table 4 FISH data for nevoid melanomas

\begin{tabular}{|c|c|c|c|c|}
\hline Subject & $\begin{array}{c}\text { RREB1/CEP6 } \\
\text { gain }\end{array}$ & $\begin{array}{l}\text { RREB1 } \\
\text { gain }\end{array}$ & $\begin{array}{c}\text { MYB/CEP6 } \\
\text { loss }\end{array}$ & $\begin{array}{l}\text { CCND1 } \\
\text { gain }\end{array}$ \\
\hline 1 & 22/30 (0.73) & $14 / 30(0.47)$ & 3/30 (0.10) & $4 / 30(0.13)$ \\
\hline 2 & $13 / 30(0.43)$ & $19 / 30(0.63)$ & $10 / 30(0.33)$ & $11 / 30(0.37)$ \\
\hline 3 & $13 / 30(0.43)$ & $29 / 30(0.97)$ & $25 / 30(0.83)$ & $23 / 30(0.77)$ \\
\hline 4 & $25 / 30(0.83)$ & $8 / 30(0.27)$ & $2 / 30(0.07)$ & $1 / 30(0.03)$ \\
\hline 5 & $17 / 30(0.57)$ & $27 / 30(0.90)$ & $7 / 30(0.23)$ & $20 / 30(0.67)$ \\
\hline 6 & 19/30 (0.63) & $25 / 30(0.83)$ & $6 / 30(0.20)$ & $9 / 30(0.30)$ \\
\hline
\end{tabular}

Bolded if met criteria.

plasms. $^{9}$ FISH represents an ideal method for analyzing melanomas with associated nevi and correlating the findings to histomorphology, thus our interest in evaluating its potential for microstaging of melanoma.

In this study, we found that gains in 6p25 (RREB1) and/or imbalance in chromosome 6 , with gains in 6p25 combined with losses in 6q23 (MYB), are a frequent finding in melanomas associated with a precursor nevus. Hence, this probe set can perform with good sensitivity in this subset of melanomas. It is not surprising that most cases tested positive secondary to an imbalance in chromosome 6 , whereas the probe for CCND1 did not play a significant role because the majority of cases in this study were from the trunk or extremities in areas of intermittently sun-exposed skin rather than from chronically sun-damaged skin. ${ }^{10}$ It is of importance to note that we did not find any significant chromosomal aberrations with this probe set in the areas that were considered benign histologically.

The test also performed well in the nevoid melanomas, accurately detecting malignancy in the deep dermal component of all six cases. This finding shows that the test has the ability to detect malignant melanocytes possessing morphological features of nevi.

On the basis of the results of this study, we believe that this probe set targeting 6p25, 6q23, 11q13 and CEP6 can assess the malignant component of a melanoma arising within a nevus with high sensitivity and the benign component with high specificity, thereby making it a useful adjunctive test for evaluating Breslow's depth. Our results suggest that any area in the dermis meeting FISH criteria for melanoma should be included in the Breslow's measurement. Importantly, in several cases in which clearly malignant changes were noted histologically however, sufficient chromosomal aberrations for a diagnosis of melanoma by FISH were not detected. Therefore, one cannot completely exclude or discount dermal nests because of a negative FISH result. However, our results from the evaluation of nevoid melanomas suggest that the assay can perform well in detecting malignant dermal melanocytes even with nevoid features. Accordingly, the FISH results should be interpreted in correlation with the histological pattern to ensure optimal diagnosis.

In conclusion, when used with histomorphological correlation, FISH analysis using probes targeting 


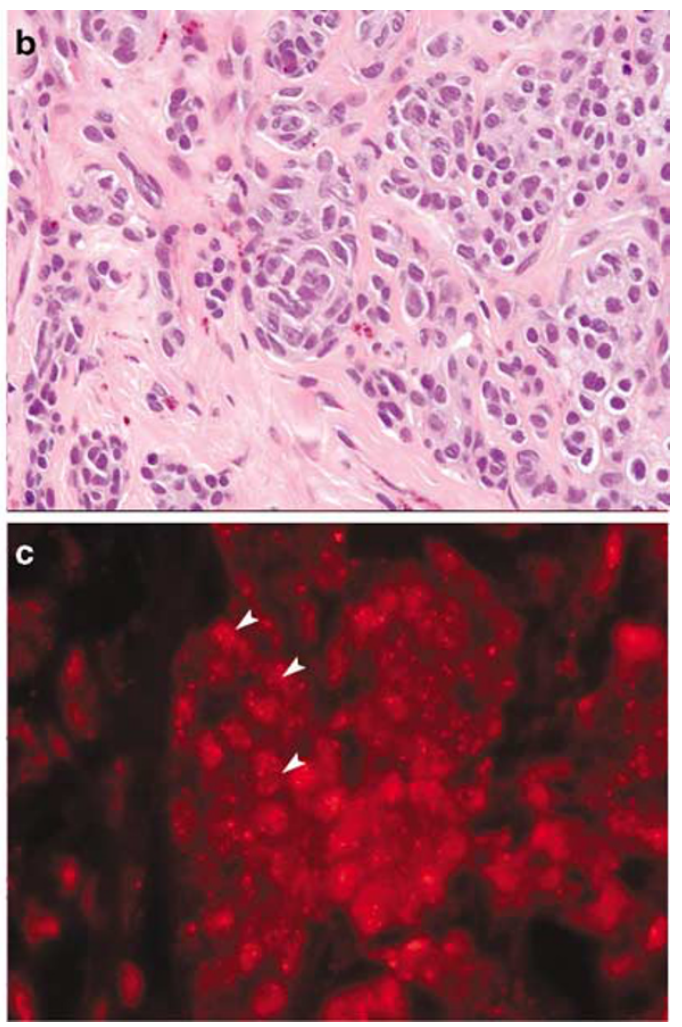

Figure 4 A representative low-power $(\mathbf{a} ; \mathrm{H} \& \mathrm{E}, \times 100)$ photograph shows atypical intermediate sized melanocytes, with a nevoid growth pattern in the dermis. At higher power, (b; H\&E $\times 400$ ), there is a focus of malignant melanocytes within the dermis corresponding to (c; FISH, $\times 600) 6 p$ chromosomal gains in the malignant cells. 
6p25, 6q23, 11q13 and CEP6 can be an extremely helpful adjunctive tool in evaluating Breslow's depth in melanomas with associated nevi. FISH results may prove particularly helpful in determining exact margins, the size of an excision in a sensitive area or the need for sentinel lymph node biopsy, all of which outweigh the cost of FISH analysis. Any dermal component that tests positive by our previously determined criteria can be definitively regarded as melanoma and included in the Breslow's measurement. However, a negative test result does not necessarily preclude that the tumor cells in question are malignant. In cases that are highly ambiguous or difficult, our recommendation is to first determine whether 6p25 gain, 6q23 loss or $11 \mathrm{q} 13$ gain is highly characteristic of the definitively malignant areas. If a characteristic chromosomal abnormality is detected in the clearly malignant area, the absence of these same changes in dermal areas would favor, but still not ensure, benignity. The use of this assay could prove extremely helpful in improving the accuracy of the Breslow's evaluation in difficult cases and establishing malignancy in the deeper dermal components of nevoid melanomas or in melanomas with pseudomaturation, which might otherwise be considered an area of precursor nevus.

\section{Conflict of interest}

Dr Gerami has served as a consultant to Abbott Molecular Labs and has received honoraria from Abbott Molecular Labs. All other authors report no relevant conflicts of interest or funding sources to declare.

\section{Acknowledgement}

This work is original and has not had prior presentation.

\section{References}

1 Bevona C, Sober AJ. Melanoma incidence trends. Dermatol Clin 2002;20:589-595, vii.

2 http://www.cancer.org/docroot/cri/content/cri_2_4_1x what_are_the_key_statistics_for_melanoma_50.asp Accessed 11-1-08.

3 Massi D, Franchi A, Borgognoni L, et al. Thin cutaneous malignant melanomas $(<$ or $=1.5 \mathrm{~mm})$ : identification of risk factors indicative of progression. Cancer 1999;85:1067-1076.

4 Bondi EE, Clark Jr WH, Elder D, et al. Topical chemotherapy of dysplastic melanocytic nevi with 5\% fluorouracil. Arch Dermatol 1981;117:89-92.

5 Williams ML, Sagebiel RW. Melanoma risk factors and atypical moles. West J Med 1994;160:343-350.

6 Skender-Kalnenas TM, English DR, Heenan PJ. Benign melanocytic lesions: risk markers or precursors of cutaneous melanoma? J Am Acad Dermatol 1995;33:1000-1007.

7 Urso C, Rongioletti F, Innocenzi D, et al. Histological features used in the diagnosis of melanoma are frequently found in benign melanocytic naevi. J Clin Pathol 2005;58:409-412.

8 Ruhoy SM, Prieto VG, Eliason SL, et al. Malignant melanoma with paradoxical maturation. Am J Surg Pathol 2000;24:1600-1614.

9 Gerami P, Jewell S, Morrison L, et al. Fluorescence in situ hybridization (FISH) as an ancillary tool in the diagnosis of melanoma. Am J Surg Pathol 2009 (in press).

10 Curtin JA, Fridlyand J, Kageshita T, et al. Distinct sets of genetic alterations in melanoma. $\mathrm{N}$ Engl J Med 2005;353:2135-2147. 